\title{
Instabilities in the winds of hot stars
}

\author{
Edward L. Gomez and Robin J.R. Williams \\ Department of Physics and Astronomy, Cardiff University, \\ 5 The Parade, Cardiff CF243YB, UK
}

\begin{abstract}
We present work investigating the stability of line-driven winds using a non-Sobolev approach. We find that the dense shell structures which characterise the growth of radiation-driven instabilities in one dimensional simulations break up into numerous bow-shaped clumps in two-dimensions.
\end{abstract}

\section{A non-Sobolev model of line-driving}

The base of a stellar wind is not uniformly smooth, as fluctuations in the properties of the stellar surface layers arise from a variety of effects. Therefore, a realistic model should account for the effect of perturbations to the mean flow.

Owocki, Castor \& Rybicki (1988) propose a pure absorption model, that dispenses with the Sobolev (large velocity gradient) approximation, using a resolved line-profile function. The acceleration of the wind is determined by an explicit calculation of the optical depth for a statistical ensemble of line opacities. This model accurately reproduces the qualitative features of more complete treatments of the unstable line-driven flows in one dimension. We have therefore adopted this model for these initial investigations of the flow structure in two dimensions.

We have used a top hat line-profile function in order to accelerate the line force calculation. As the absorption by gas within a single computational cell is localized in frequency, only a small range of frequency bins needs to be included in the calculation of the radiation force on the cell and the change in the transmitted spectrum. This local approach allows the computational expense to be greatly reduced (particularly important for multidimensional simulations).

In one dimension, we find results similar to Owocki et al. (1988). Using a smooth CAK (Castor, Abbott \& Klein 1975) flow as the initial condition, we find that dense shells arise from the growth of small numerical fluctuations at the wind base. When a finite perturbation is applied to the initial flow (in either density or velocity), it grows into a rarefication wave leading to a plane reverse shock. Eventually, the shock becomes unstable and sends dense lumps into the trailing rarefaction. Once these structures have left the grid, the flow returns to a structure similar to that in the unperturbed case.

In 2D slab symmetry, the initial growth of a perturbation is similar to that in a 2D calculation using the Sobolev approximation. However instabilities soon become important in the non-Sobolev model and the evolving perturbation is disrupted. As the perturbation increases in amplitude, the radiation driving of 


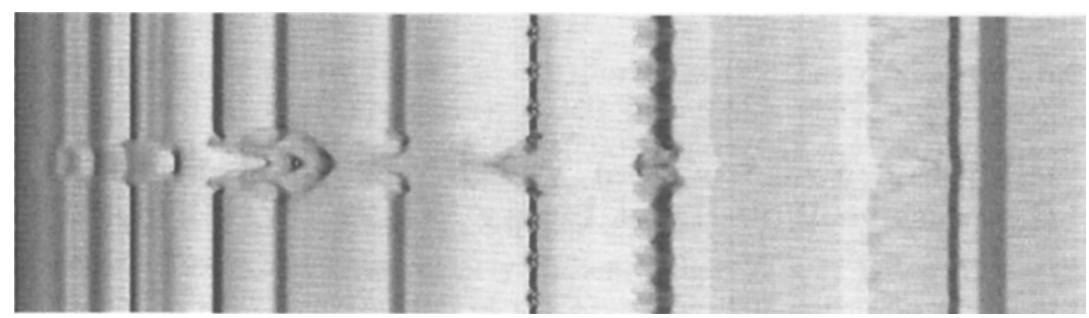

Figure 1. The flow density in the expansion of a small perturbation a line driven wind in a box $1 R_{*}$ tall that extends from $1-4 R_{*}$. Variations can be seen in the shadow of the initial perturbation, while away from the shadow, thin shell instabilities can be seen forming. This was calculated on a fixed grid of $800 \times 400$ cells, using a modified version of the hydrodynamical code VH-1 (Blondin 1990). The maximum density is $\sim 2 \times 10^{-11} \mathrm{~g} \mathrm{~cm}^{-3}$.

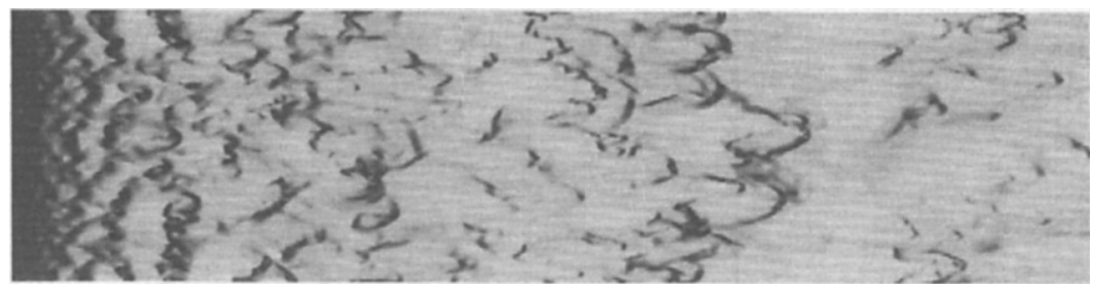

Figure 2. The same piece of photosphere at a later time. Note that while the flow can still be characterised as the ejection of successive shells of dense material, the shells are highly fragmented and limited in extent.

the gas downstream varies, with gas decelerating where the relevant region of the spectrum is now absorbed and accelerating where the upstream absorption has decreased. The resulting velocity perturbations form bow shocks as they grow. Away from the shadow of the initial perturbation, thin shell instabilities are triggered and also grow to form bow shocks. While the bow shocks initially form only on the high density shells, with time the shells break up entirely and bow shock structures are found throughout the flow. Eventually all obvious direct effects of the initial perturbation are advected from the grid, but residual numerical perturbations at the base of the wind continue to drive instabilities leaving a highly structured flow.

Much additional physics remains to be included in these models, but it seems likely the overall properties of the line driven instabilities will remain similar to those we present here. Could this be a glimpse of the structure of the winds of OB stars?

\section{References}

Blondin, J.M., Kallman, T.R., Fryzell, B.A., Taam, R.E. 1990 ApJ 356, 591

Castor, J.I., Abbott, D.C., Klein, R.I. 1975 ApJ 195, 157

Owocki, S.P., Castor, J.I., Rybicki, G.B. 1988, ApJ 335, 914 